Colleen G. Lance, MD

Sleep Disorders Center, Neurological Institute,

Cleveland Clinic

\title{
PAP therapy increases the risk of transmission of COVID-19
}

\section{Posted April 8, 2020}

\begin{abstract}
Positive airway pressure (PAP) therapy is an open system that can generate contact, droplet, and airborne COVID-19 thereby increasing the risk of transmission of COVID-19 like other procedures such as tracheal intubation, noninvasive ventilation, and cardiopulmonary resuscitation. Medically prudent recommendations have been established for home use of PAP therapy in asymptomatic patients and patients with suspected or confirmed COVID-19. Recommendations for sleep clinics and sleep laboratory activities are also discussed as well as the emergency use of PAP devices as a substitute for ventilation.
\end{abstract}

\section{INTRODUCTION}

All forms of positive airway pressure (PAP) therapy should be assumed to increase the risk of droplet and aerosolized transmission of COVID-19.1,2 PAP therapy is an open system that can generate contact, droplet, and airborne COVID-19, increasing the risk of transmission like other procedures such as tracheal intubation, noninvasive ventilation, and cardiopulmonary resuscitation. ${ }^{3}$ The COVID-19 virus is also known to be viable for several hours after aerosolization. ${ }^{4}$ Empirical contact, droplet, and airborne precautions are necessary for patients on PAP therapy in the context of the COVID-19 pandemic.

Medically prudent recommendations are available from the American Academy of Sleep Medicine (aasm. org/covid-19-resources/covid-19-faq) (AASM) and from other experts on the COVID-19 blog post (aasm.org/ covid-19-information-for-sleep-medicine-clinicians). The major recommendations are summarized here.

The statements and opinions expressed in COVID-19 Curbside Consults are based on experience and the available literature as of the date posted. While we try to regularly update this content, any offered recommendations cannot be substituted for the clinical judgment of clinicians caring for individual patients.

doi:10.3949/ccjm.87a.ccc003
PAP FOR THE ASYMPTOMATIC PATIENT AT HOME

- Use of PAP therapy may increase the risk of transmission of COVID-19 to others in the environment (ie, cohabitants in the same dwelling).

- The risk of re-infection from tubing, filters, or mask reuse is not known.

- The PAP therapy user should sleep in a separate bedroom and if possible, use a separate bathroom.

- Diligently cleaning PAP equipment according to manufacturer's guidelines (eg, Philips [www.usa. philips.com/c-e/hs/better-sleep-breathing-blog/better-sleep/keeping-it-clean-cpap.html], ResMed [www. resmed.com/en-us/sleep-apnea/cpap-parts-support/ cleaning-cpap-equipment/]).

- Use distilled water in the humidifier (how to make distilled water [www.wikihow.com/MakeDistilled-Water]). If distilled water is not available, short-term use of purified bottled water or filtered tap water is acceptable. Avoid use of well water. Replacement humidification chambers are available from durable medical equipment companies.

- Completely dry the humidifier, mask, and tubing after it is washed.

- Disinfect the humidifier chamber per the manufacturer's guidelines. Generally, a vinegar and water solution is suitable. Do not use chemicals or disinfectants in the humidifier chamber that are not recommended by the manufacturer, which can lead to inhalational lung injury.

- Avoid leaving the PAP device on when not in use.

- Address significant mask leaks with the medical equipment company.

\section{PAP FOR THE PATIENT WITH SUSPECTED OR CONFIRMED OF COVID-19}

- If a patient uses PAP therapy for the treatment of obstructive sleep apnea, it may be reasonable to discontinue PAP therapy for a short duration until the patient is no longer contagious. The patient 
should be counseled about the risks of the short-term discontinuation of PAP therapy, such as sleepinessrelated accidents or cardiovascular events. It would be reasonable to suggest the following interim measures:

- Positional therapy with side sleeping, or head elevated at least 30 degrees

- Use of a dental appliance if the patient already has one

- Treatment of nasal congestion

- Avoidance of alcohol and sedating medications

- Avoidance of drowsy driving.

- If the risk of suspending PAP therapy is too great, the patient should be advised to maintain strict quarantine and consider strategies for protecting household contacts.

- If the patient is admitted to the hospital for any reason, advise them to bring their PAP equipment with them.

- Once the patient is noninfectious, changing all PAP therapy disposables such as the mask, tubing, and filters is recommended.

\section{SLEEP CLINICS AND SLEEP LABORATORY ACTIVITIES}

The AASM has issued recommendations about sleep clinic and sleep laboratory activities (aasm.org/covid19-resources/covid-19-mitigation-strategies-sleepclinics-labs ) during substantial community spread of COVID-19. To briefly summarize:

- Postpone in-laboratory sleep studies for all patients. Cleveland Clinic evaluates potentially urgent requests on a case-by-case basis.

- Postpone home sleep apnea testing unless using only disposable testing devices. Cleveland Clinic uses disposable parts and disinfects nondisposable components.

- Sleep clinics should remain open for phone calls, telemedicine visits, and emergency in-person visits only.

- Communicate with patients and other stakeholders (eg, payors, durable medical equipment companies, trucking companies) about the need to extend deadlines for the completion of sleep study evaluation and follow-up visits.

- The AASM is advocating to relax payor requirements during this national emergency:

- Currently, the Centers for Medicare \& Medicaid Services (CMS) is relaxing the required face-to-face or in-person encounters for evaluations, assessments, and certifications.

- In addition, during the pandemic CMS is allowing coverage of PAP therapy based on a clinician's assessment.

- Telehealth modalities that include audio and video 2 -way communications between patient and provider are accepted.

- Penalties are being waived for Health Insurance Portability and Accountability Act privacy violations associated with the use of FaceTime or Skype.

\section{EMERGENCY USE OF PAP DEVICES AS A SUBSTITUTE FOR VENTILATION}

Basic continuous PAP and bilevel PAP devices are not designed to function as ventilators. Adaptive servo ventilation is a device specifically used to treat central sleep apnea and is also not meant to function as a ventilator in the scenario of respiratory failure.

PAP therapy devices with noninvasive ventilation (NIV) capacity include:

- Bilevel PAP ST (spontaneous timed)

- AVAPS (average volume-assured pressure support)

- iVAPS (intelligent volume-assured pressure support).

These devices are not a substitute for the use of a ventilator in the setting of acute respiratory failure. Importantly, these devices are also open circuits with the same risk of droplet and aerosolized particle spread of virus as other PAP devices. There is recent history of using NIV in critically ill patients due to Middle East respiratory syndrome in a retrospective cohort study. Results were that NIV failure was high and not associated with improved outcomes. ${ }^{5}$

Should the need arise to use PAP/NIV devices to assist with mild hypoxemia and hypercarbia pending endotracheal intubation, adaptations will need to be made to the mask and filtration systems. In these scenarios, it is best to use an oronasal mask that is well fitted and avoid nasal masks or nasal pillows. New information is emerging daily in this area. Monitoring manufacturers' (www.philips.com/a-w/about/news/ archive/blogs/innovation-matters/2020/20200325covid-19-how-to-safely-optimize-niv-therapy.html) and medical societies is recommended: American Academy of Sleep Medicine (AASM, aasm.org), American Society of Anesthesiologists (ASA, www. asahq.org), American College of Chest Physicians (CHEST, www.chestnet.org), and the American Thoracic Society (ATS, www.thoracic.org).

\section{REFERENCES}

1. Singh A, Singh J. Noninvasive ventilation in acute respiratory failure due to H1N1 influenza: A word of caution. Lung India 2011; 
28(2):151. doi:10.4103/0970-2113.80340

2. Tang JW, Li Y, Eames I, Chan PK, Ridgway GL. Factors involved in the aerosol transmission of infection and control of ventilation in healthcare premises. J Hosp Infect 2006; 64(2):100-114. doi:10.1016/j.jhin.2006.05.022

2. Hui DS. Epidemic and Emerging Coronaviruses (Severe Acute Respiratory Syndrome and Middle East Respiratory Syndrome). Clin Chest Med 2017; 38(1):71-86. doi:10.1016/j.ccm.2016.11.007
4. van Doremalen N, Bushmaker T, Morris DH, et al. Aerosol and Surface Stability of SARS-CoV-2 as Compared with SARS-CoV-1 [published online ahead of print, 2020 Mar 17]. N Engl J Med 2020; NEJMc2004973. doi:10.1056/NEJMc2004973

5. Alraddadi BM, Qushmaq I, Al-Hameed FM, et al. Noninvasive ventilation in critically ill patients with the Middle East respiratory syndrome. Influenza Other Respir Viruses 2019; 13(4):382-390. doi:10.1111/irv.12635 